PROCEEDINGS OF THE

AMERICAN MATHEMATICAL SOCIETY

Volume 134, Number 1, Pages 273-282

S 0002-9939(05)08233-X

Article electronically published on August 15, 2005

\title{
THE BIG SLICE PHENOMENA IN M-EMBEDDED AND L-EMBEDDED SPACES
}

\author{
GINÉS LÓPEZ PÉREZ
}

(Communicated by N. Tomczak-Jaegermann)

\begin{abstract}
We obtain sufficient conditions on an M-embedded or L-embedded space so that every nonempty relatively weakly open subset of its unit ball has norm diameter 2. We prove that, up to renorming, this holds for every Banach space containing $c_{0}$ and, as a consequence, for every proper M-ideal. The result obtained for L-embedded spaces can be applied to show that the above property is satisfied for every predual of an atomless real JBW*triple. As a consequence, a characterization of the Radon-Nikodym property is obtained in this setting, showing that a predual of a real $\mathrm{JBW}^{*}$-triple $\mathrm{E}$ verifies the Radon-Nikodym property if, and only if, $\mathrm{E}$ is the $l_{\infty}$-sum of real type I triple factors.
\end{abstract}

\section{INTRODUCTION}

The nonexistence of denting points in the unit ball of some function spaces has been the subject of several recent papers [13, 23]. A point $x_{0}$ in the sphere of a Banach space $X, S_{X}$, is a denting point of the unit ball in $X, B_{X}$, if there are slices, that is, subsets defined as

$$
S\left(x^{*}, \alpha\right)=\left\{x \in B_{X}: x^{*}(x)>\left\|x^{*}\right\|-\alpha\right\}, x^{*} \in X^{*}, \alpha \in \mathbb{R},
$$

containing $x_{0}$, with diameter arbitrarily small. From [6], $x_{0}$ is a denting point of the unit ball of $X$ if, and only if, $x_{0}$ is an extreme point in $B_{X}$ and $x_{0}$ is a point of weak-norm continuity, that is, a point of continuity for the identity map from $\left(B_{X}, w\right)$ onto $\left(B_{X}, n\right)$, where $w$ and $n$ denote the weak and the norm topology, respectively. In particular, the existence of denting points in the unit ball of a Banach space $X$ implies the existence of nonempty relatively weakly open subsets of the unit ball in $X$ with diameter arbitrarily small. Then the extreme opposite property to the existence of denting points in the unit ball of a Banach space is that every nonempty relatively weakly open subset of the unit ball has diameter 2. This is the case, for example, for infinite-dimensional $C^{*}$-algebras [5], uniform algebras [20, non-hilbertizable real JB*-triples [4] and for some Banach spaces of vector valued functions and some spaces of operators 3 .

The aim of this note is to study when every nonempty relatively weakly open subset of the unit ball of an M-embedded or L-embedded space has diameter 2. In Theorem 2.4, we obtain sufficient conditions in order to assure the above property

Received by the editors September 8, 2004.

2000 Mathematics Subject Classification. Primary 46B20, 46B22.

Key words and phrases. Denting point, slices, weakopen subsets.

(C)2005 American Mathematical Society Reverts to public domain 28 years from publication 
in the M-ideals case, when only the original norms are considered, by improving the results in [23]. After this, it is shown in Proposition 2.6 that every Banach space containing $c_{0}$ can be equivalently renormed so that every nonempty relatively weakly open subset of its unit ball has diameter 2, and then the same is true for proper M-ideals.

The result for the L-embedded case is Theorem 2.8, where a sufficient condition to have diameter 2 for all nonempty relatively weakly open subsets of the unit ball of an L-embedded space is obtained. This condition works in the setting of preduals of real $\mathrm{JBW}^{*}$-triples and, as a consequence, we prove in Theorem 2.12 that every nonempty relatively weakly open subset of the unit ball of the predual of an atomless real $\mathrm{JBW}^{*}$-triple has diameter 2 . Then the same holds for preduals of atomless Von Neumann algebras. Finally an easy characterization of the RadonNikodym property is given in Theorem 2.14, where it is shown that the predual of a real $\mathrm{JBW}^{*}$-triple $E$ satisfies the Radon-Nikodym property if, and only if, $E$ is the $l_{\infty}$-sum of type I real triple factors and then, the predual $B$ of a Von Neumann algebra $A$ satisfies the Radon-Nikodym property if, and only if, $B$ is the $c_{0}$-sum of trace class operators on a complex Hilbert space. The above characterizations of Radon-Nikodym property can be found in 8 and 2 for preduals of complex $\mathrm{JBW}^{*}$-triples and in 9] for preduals of Von Neumann algebras. Also the relation between L-embedded spaces and the Radon-Nikodym propety was studied in [17.

Finally, we also apply Theorem 2.8 in order to obtain that every nonempty relatively weakly open subset of the unit ball of $L_{1} / H^{1}$ has diameter 2 , and Corollary 2.9, where $L_{1}$ and $H^{1}$ denote the classical Lebesgue space and Hardy space, respectively, on the unit interval $[0,1]$.

Let $X$ be a real or complex Banach space. We denote by $S_{X}, B_{X}$, and $X^{*}$ the unit sphere, the closed unit ball and the topological dual, respectively, of $X$. We denote by $w$ the weak topology of $X$, and by $w^{*}$ the weak* topology of $X^{*}$. Given a subspace $M$ of $X$, we denote by $M^{\circ}$ the polar or annihilator subspace of $M$ in $X^{*}$. An L-projection (resp. M-projection) on $X$ is a linear projection $p$ on $X$ satisfying $\|x\|=\|p(x)\|+\|x-p(x)\|$ (resp. $\|x\|=\max \{\|p(x)\|,\|x-p(x)\|\}$ ) for all $x \in X$. A subspace $M$ of $X$ is said to be an L-summand (resp. M-summand) of $X$ if it is the range of an L-projection (resp. M-projection) on $X$, and an M-ideal of $X$ if $M^{\circ}$ is an L-summand of $X^{*}$. $X$ is said to be L-embedded (resp. M-embedded) whenever $X$ is an L-summand (resp. M-ideal) of $X^{* *}$ (see [12]).

\section{The Main Results}

We begin with an elementary lemma which will be essential for the main results.

Lemma 2.1. Let $X$ be a Banach space such that every nonempty relatively weakly open subset of $B_{X}$ has diameter 2 . Then every nonempty relatively weakly open subset of $B_{X \oplus_{\infty} Y}$ has diameter 2 , where $Y$ is an arbitrary Banach space.

Proof. We call $Z=X \oplus_{\infty} Y$ and let $P: Z \rightarrow X$ be the projection from $Z$ onto $X$, which is weak open. It is clear that $B_{Z}=B_{X} \times B_{Y}$ and $\|P\|=1$. Then if $W$ is a weakly open subset of $Z$ such that $W \cap B_{Z} \neq \emptyset$, one has that $V=P\left(W \cap B_{Z}\right)$ is a nonempty weak open set relative to $B_{X}$, and so $\operatorname{diam}(V)=2$. Hence $\operatorname{diam}\left(W \cap B_{Z}\right)=2$.

The following is a $w^{*}$ version of the above lemma. We omit the proof, since it is similar to the one above. 
Lemma 2.2. Let $X$ be a Banach space. Assume that $X^{* *}=Y^{\circ \circ} \oplus_{\infty} Z^{\circ}$, where $Y$ is a closed subspace of $X$ and $Z$ is a closed subspace of $X^{*}$. Assume that every nonempty relatively $w^{*}\left(Y^{\circ \circ}\right)$-open subset of $B_{Y^{\circ \circ}}$ has diameter 2 . Then every nonempty relatively $w^{*}\left(X^{* *}\right)$-open subset of $B_{X^{* *}}$ has diameter 2 .

For a Banach space $X$ and a subspace $Z$ of $X^{*}$ given, we denote by $\sigma(X, Z)$ the weak topology on $X$ endowed by the dual pair $(X, Z)$, that is, the smallest vector topology on $X$ such that every element of $Z$ is a continuous map.

The following result shows that the size of many nonempty relatively weakly open subsets of the unit ball of an M-ideal have diameter 2 .

Proposition 2.3. Let $X$ be a Banach space and let $Y$ be a closed and proper subspace of $X$. Assume that $Y$ is an $M$-ideal of $X$ (that is, there is an L-projection from $X^{*}$ onto some subspace $Z$ of $X^{*}$, with kernel $\left.Y^{\circ}\right)$. Then every nonempty relatively $\sigma(X, Z)$-open subset of $B_{X}$ which intersects $B_{Y}$ has diameter 2.

Proof. Let $U$ be a $\sigma(X, Z)$-open subset of $X$ and assume that $U \cap B_{Y} \neq \emptyset$. Choose some $x_{0} \in U \cap B_{Y}$.

As $Y$ is a proper subspace of $X$, given $\varepsilon>0$, there is an $x \in S_{X}$ such that $\|x+Y\|>1-\varepsilon$, where $x+Y$ denotes the class of the element $x$ in the quotient $X / Y$.

By [22, Proposition 2.3], there exists a net $\left\{x_{\alpha}\right\}$ of elements of $Y$ converging to $x$ in the $\sigma(X, Z)$-topology and satisfying

$$
\limsup _{\alpha}\left\|x_{0} \pm\left(x-x_{\alpha}\right)\right\| \leq 1 .
$$

Then, for $0<\lambda<1$ given, we can choose $\alpha_{0}$ so that $\lambda\left\|x_{0} \pm\left(x-x_{\alpha}\right)\right\| \leq 1$, whenever $\alpha \geq \alpha_{0}$.

Furthermore, for $\lambda$ close enough to 1 , we can assume that $\lambda\left(x_{0} \pm\left(x-x_{\alpha}\right) \in U\right.$ for each $\alpha \geq \alpha_{0}$, since the net $\left\{\lambda\left(x_{0} \pm\left(x-x_{\alpha}\right)\right)\right\}$ converges to $\lambda x_{0}$ in the $\sigma(X, Z)$-topology and $x_{0} \in U \cap S_{Y}$.

Then $\lambda\left(x_{0} \pm\left(x-x_{\alpha}\right)\right) \in U \cap B_{X}$, whenever $\alpha \geq \alpha_{0}$ and $0<\lambda<1$ is close enough to 1 . Hence

$$
\operatorname{diam}\left(U \cap B_{X}\right) \geq 2 \lambda\left\|x-x_{\alpha}\right\| \geq 2 \lambda\|x+Y\|>2 \lambda(1-\varepsilon)
$$

whenever $\alpha \geq \alpha_{0}$ and $0<\lambda<1$ are close enough to 1 . Now, it is enough to take the limit when $\lambda$ tends to 1 and $\varepsilon$ to 0 , to obtain that $U$ has diameter 2 .

The following is the main result in the M-ideals setting, which improves the results in 23, where only the nonexistence of strongly exposed points is deduced with an extra hypothesis.

Theorem 2.4. Let $X$ be a Banach space and let $Y$ be a closed and proper subspace of $X$. Assume that $Y$ is an $M$-ideal of $X$ (that is, there is an L-projection on $X^{*}$ onto some subspace $Z$ of $X^{*}$, with kernel $\left.Y^{\circ}\right)$. If $B_{Z}$ is weak-* dense in $B_{X^{*}}$, then every nonempty relatively weakly open subset of $B_{X}$ and $B_{Y}$ has diameter 2 .

Proof. As $B_{Z}$ is weak-* dense in $B_{X^{*}}$, then $\|x\|=\sup _{z \in B_{Z}}|z(x)| \forall x \in X$, and so the norm of $X$ is $\sigma(X, Z)$-lower semi-continuous.

Let $U$ be a nonempty relatively weakly open subset of $B_{Y}$. Then, there are $z_{1}, \cdots, z_{n} \in Z$ and $y_{0} \in B_{Y}$ such that

$$
U_{0}=\left\{y \in B_{Y}:\left|z_{i}\left(y-y_{0}\right)\right|<1,1 \leq i \leq n\right\} \subset U,
$$


since the $\sigma(X, Z)$-topology on $Y$ is just the weak topology of $Y$.

Setting $V=\left\{x \in B_{X}:\left|z_{i}\left(x-y_{0}\right)\right|<1,1 \leq i \leq n\right\}$, we have that $V$ is a nonempty relatively $\sigma(X, Z)$ open subset of $B_{X}$ intersecting $B_{Y}$. By Proposition 2.3, we obtain that $\operatorname{diam}(V)=2$. Now, we claim that $U_{0}$ is dense in the topological space $(V, \sigma(X, Z))$.

Indeed, $X^{* *}=Y^{\circ \circ} \oplus_{\infty} Z^{\circ}$; hence every $x \in B_{X}$ can be written as $x=u+v$ with $u \in B_{Y \circ \circ}$ and $v \in B_{Z^{\circ}}$. There exists a net of elements $y_{\alpha} \in B_{Y}$ which converges to $u$ in the weak-* topology. Hence, for every $z \in Z$, we have $z(x)=(u+v)(z)=$ $u(z)=\lim _{\alpha} z\left(y_{\alpha}\right)$. Now it is clear that the assumption $\left|z_{i}\left(x-y_{0}\right)\right|<1,1 \leq i \leq n$, implies that, for some $\alpha_{0}$ and $\alpha \geq \alpha_{0},\left|z_{i}\left(y_{\alpha}-y_{0}\right)\right|<1,1 \leq i \leq n$. That proves the $\sigma(X, Z)$-density of $U_{0}$ in $V$. Moreover, as the norm of $X$ is $\sigma(X, Z)$-lower semicontinuous, we have that $\operatorname{diam}\left(U_{0}\right)=2$ and so, $\operatorname{diam}(U)=2$. Then we have proved that every nonempty relatively weakly open subset of $B_{Y}$ has diameter 2 .

As every nonempty relatively weak-* open subset of $B_{Y^{* *}}$ contains a nonempty relatively weakly open subset of $B_{Y}$, and $B_{Y}$ is weak-* dense in $B_{Y^{* *}}$, we deduce that every nonempty relatively weak-* open subset of $B_{Y^{* *}}$ also has diameter 2. Now, as $Y$ is an M-ideal of $X$, we have $X^{*}=Y^{\circ} \oplus_{1} Z$ and then $X^{* *}=Y^{\circ \circ} \oplus_{\infty} Z^{\circ}$, where, $\oplus_{1}$ and $\oplus_{\infty}$ denote the $\ell_{1}$ and $\ell_{\infty}$ sum, respectively. Hence, by Lemma 2.2, we have that every nonempty relatively weak-* open subset of $B_{X^{* *}}$ has diameter 2 , and then every nonempty relatively weakly open subset of $B_{X}$ also has diameter 2 , since from the weak-* density of $B_{X}$ in $B_{X^{* *}}$, every nonempty relatively weakly open subset of $B_{X}$ is weak-* dense in some nonempty relatively weak-* open subset of $B_{X^{* *}}$ and the norm in $X^{* *}$ is weak-* lower semi-continuous.

For M-embedded Banach spaces $X$, we have $X^{* * *}=X^{*} \oplus_{1} X^{\circ}$, and so we have automatically the weak-* density of $B_{X^{*}}$ in $B_{X^{* * *}}$. Then we obtain the following

Corollary 2.5. Let $X$ be a nonreflexive $M$-embedded Banach space, and let $Y$ be a closed subspace of $X^{* *}$ containing $X$. Then every nonempty relatively weakly open subset of $B_{Y}$ has diameter 2 .

Proof. If $Y$ is a closed subspace of $X^{* *}$ containing $X$, then $X$ is an M-ideal of $Y$. In fact, if $p$ is the L-projection in $X^{* * *}$ with kernel $X^{\circ}$ and image $X^{*}$, we identify $Y^{*}$ with the quotient $X^{* * *} / Y^{\circ}$ and define $\pi: Y^{*} \rightarrow Y^{*}$ by $\pi\left(x^{* * *}+Y^{\circ}\right)=p\left(x^{* * *}\right)+Y^{\circ}$. Now $\pi$ is an L-projection whose kernel is the anihilator of $X$ in $Y^{*}$.

Finally, as $B_{X^{*}}$ is weak-* dense in $B_{X^{* * *}}$, given $x^{* * *} \in X^{* * *}$ with $\left\|x^{* * *}+Y^{\circ}\right\| \leq$ 1 , we choose $y^{\circ} \in Y^{\circ}$ such that $\left\|x^{* * *}+y^{\circ}\right\| \leq 1$, and then there exists a net $\left\{z_{\lambda}\right\}$ of elements of $B_{X^{*}}$ converging to $x^{* * *}+y^{\circ}$ in the weak-* topology on $X^{* * *}$. Then $\left\{z_{\lambda}+Y^{\circ}\right\}$ is a net of elements of $B_{\pi\left(Y^{*}\right)}$ converging to $x^{* * *}+Y^{\circ}$ in the weak-* topology on $Y^{*}$. Then we have proved that the closed unit ball of $\pi\left(Y^{*}\right)$ is weak-* dense in $B_{Y^{*}}$. It is enough to apply Theorem 2.4 to obtain that every nonempty relatively weakly open subset of $B_{Y}$ has diameter 2 .

Note that, since the property of being M-embedded is hereditary and stable by quotients, the same result is true when $Y$ is a nonreflexive closed subspace of $X$ or a nonreflexive quotient of $X$.

In particular, from the above corollary, we deduce that the unit closed ball of every closed and nonreflexive subspace of an M-embedded space has no continuity points and so has no strongly exposed points, and the same is true for nonreflexive quotients of an M-embedded spaces. Roughly speaking, this fact shows 
that every subspace and every quotient of an M-embedded space fails the RadonNikodym property in a very strong way, if it is not reflexive. As a consequence of the above corollary, it is worth mentioning some interesting examples. As $c_{0}$ is an M-embedded space, not only every infinite-dimensional subspace or quotient of $c_{0}$ verifies the conclusion of Corollary 2.5, but also every subspace of $\ell_{\infty}$ containing $c_{0}$. If $H$ is a Hilbert space, and $K(H)$ and $L(H)$ stand for the space of compact operators on $H$ and the space of all bounded operators on $H$, respectively, it is well known that $K(H)$ is an M-embedded space. Again, not only every subspace or quotient of $K(H)$ satisfies the conclusion of Corollary 2.5. but also every subspace of $L(H)$ containing $K(H)$, since $K(H)^{* *}$, the bidual space of $K(H)$, is isometrically isomorphic to $L(H)$.

As the failure of the Radon-Nikodym property in nonreflexive M-embedded spaces is well known, since every nonreflexive M-embedded space contains an isomorphic copy of $c_{0}$, it is natural to ask for the behavior of relatively weakly open subsets of the unit ball of a Banach space containing $c_{0}$-copies. Of course, not every Banach space containing $c_{0}$-copies lacks a point of continuity in its unit ball. For this, it is enough to consider $X=c_{0} \oplus_{1} \ell_{1}$. It is easy to see that $\left(0, e_{1}\right)$ is a denting point of $B_{X}$, where $e_{1}$ denotes the first vector of the unit vector basis in $\ell_{1}$. However the following result shows that, up to renorming, the above situation cannot happen.

Proposition 2.6. Let $X$ be a Banach space containing a subspace isomorphic to $c_{0}$. Then there exists an equivalent norm in $X$ so that every nonempty relatively weakly open subset of the new unit ball has diameter 2 .

Proof. As the conclusion is isomorphic in nature, we can suppose that $X$ contains a subspace $Y$ isometric to $c_{0}$. Now, by [12, Proposition II.2.10], there exists an equivalent norm || on $X$ which agrees with the original norm on $Y$ so that $Y$ becomes M-ideal in $X$.

Then we have $\left(X,||^{*}\right)^{* *}=Z \oplus_{\infty} Y^{\circ \circ}$ for some subspace $Z$ of $X^{* *}$. Finally, as $Y^{\circ \circ}$ is isometric to $\ell_{\infty}$ and every nonempty relatively weakly open subset of $B_{\ell_{\infty}}$ has diameter 2 , it is enough to apply Lemma 2.1 to obtain that every nonempty relatively weakly open subset of $\left(B_{X},||\right)$ has diameter 2 .

As every proper M-ideal, that is, an M-ideal which is not an M-summand, contains an isomorphic copy of $c_{0}$, we deduce the following

Corollary 2.7. Let $X$ be a proper $M$-ideal of a superspace $Y$. Then there is an equivalent norm in $Y$ so that every nonempty relatively weakly open subset of the new unit ball of $X$ and $Y$ has diameter 2 .

Now we pass to study the size of nonempty relatively weakly open subsets of the unit ball of an L-embedded space. The result is the following

Theorem 2.8. Let $X$ be an L-embedded Banach space, that is, $X^{* *}=X \oplus_{1} Z$ for some subspace $Z$ of $X^{* *}$. If $B_{Z}$ is weak-* dense in $B_{X^{* *}}$, then every nonempty relatively weakly open subset of $B_{X}$ has diameter 2 .

Proof. Let $U$ be a nonempty relatively weakly open subset of $B_{X}$. As $X$ is infinite dimensional, there is an $x_{0} \in U \cap S_{X}$ and then there exist $f_{1}, \cdots, f_{n} \in X^{*}$ such that

$$
U_{0}=\left\{x \in B_{X}:\left|f_{i}\left(x-x_{0}\right)\right|<1,1 \leq i \leq n\right\} \subset U
$$


Let $V=\left\{x^{* *} \in B_{X^{* *}}:\left|f_{i}\left(x^{* *}-x_{0}\right)\right|<1,1 \leq i \leq n\right\}$. Then $V$ is a nonempty relatively weak-* open subset of $B_{X^{* *}}$ such that $x_{0} \in U_{0} \subset V$.

From the weak-* density of $B_{Z}$ in $B_{X^{* *}}$ we can choose a net $\left\{z_{\lambda}\right\}$ of elements in $B_{Z}$ converging to $x_{0}$ in the weak-* topology of $X^{* *}$, hence there is a $\lambda_{0}$ such that $z_{\lambda} \in V$, whenever $\lambda \geq \lambda_{0}$. Furthermore, as the norm of $X^{* *}$ is weak-* lower semi-continuous, we have $\liminf _{\lambda}\left\|z_{\lambda}\right\| \geq\left\|x_{0}\right\|=1$. Then, given $\varepsilon>0$ then there is a $\mu \geq \lambda_{0}$ such that $\left\|z_{\mu}\right\|>1-\varepsilon$, and $z_{\mu} \in V$. Now, we deduce

$$
\operatorname{diam}(V) \geq\left\|x_{0}-z_{\mu}\right\|=\left\|x_{0}\right\|+\left\|z_{\mu}\right\|>1+1-\varepsilon=2-\varepsilon .
$$

As $\varepsilon>0$ was arbitrary, we conclude that $\operatorname{diam}(V)=2$.

Finally, from the weak-* density of $B_{X}$ in $B_{X^{* *}}$ we obtain that $U_{0}$ is relatively dense in the topological space $V$ endowed with the weak-* topology of $X^{* *}$. Now, the weak-* lower semicontinuity of the norm in $X^{* *}$ allows us to assure that $\operatorname{diam}(U) \geq \operatorname{diam}\left(U_{0}\right)=\operatorname{diam}(V)=2$.

In order to show examples where Theorem 2.8 works, we denote by $H^{1}$ and $L_{1}$ the Hardy space and the Lebesgue space on the interval [0,1]. Also, $H_{0}^{1}$ stands for the subspace of $H^{1}$ of functions in $H^{1}$ vanishing at 0 . From [1, p. 27], the unit ball of $L_{1} / H_{0}^{1}$ lacks extreme points, and it is well known that $L_{1} / H_{0}^{1}$ is an L-embedded space. Then we can apply Theorem 2.8 as in Theorem 2.12 to obtain the following

Corollary 2.9. Every nonempty relatively weakly open subset of the unit ball of $L_{1} / H_{0}^{1} h$ as diameter 2 .

The same is true for $L_{1} / H^{1}$ instead $L_{1} / H_{0}^{1}$ since they are isometric.

Let $X$ be a Banach space. For $u$ in $S_{X}$, we define the roughness of $X$ at $u$, $\eta(X, u)$, by the equality

$$
\eta(X, u):=\lim \sup _{\|h\| \rightarrow 0} \frac{\|u+h\|+\|u-h\|-2}{\|h\|} .
$$

We remark that the absence of roughness of $X$ at $u$ (i.e., $\eta(X, u)=0$ ) is nothing other than the Fréchet differentiability of the norm of $X$ at $u$ [10, Lemma I.1.3]. Given $\epsilon>0$, the Banach space $X$ is said to be $\epsilon$-rough if, for every $u$ in $S_{X}$, we have $\eta(X, u) \geq \epsilon$. We say that $X$ is rough whenever it is $\epsilon$-rough for some $\epsilon>0$, and extremely rough whenever it is 2-rough.

Assume that $X$ is a Banach space such that every nonempty relatively weakly open subset of $B_{X}$ has diameter 2. Then, by [10, Proposition I.1.11], the dual $X^{*}$ of $X$ (resp. the predual $X_{*}$ of $X$, if this exists) is extremely rough.

Then, we have the following consequences (see Theorem 2.12 and Corollary 2.13 for part ii)):

Corollary 2.10. The following Banach spaces are extremely rough:

i) The dual of any nonreflexive $M$-embedded space.

ii) The real atomless $J B W^{*}$-triples, and so the atomless Von Neumann algebras.

Corollary 2.11. Every Banach space $X$ containing an isomorphic copy of $c_{0}$ can be equivalently renormed so that $X^{*}$ becomes extremely rough.

In order to show a new application of Theorem 2.8 we introduce some notation.

We recall that a complex $\mathrm{JB}^{*}$-triple is a complex Banach space $X$ with a continuous triple product $\{\cdots\}: X \times X \times X \rightarrow X$ which is linear and symmetric in 
the outer variables and conjugate-linear in the middle variable, and satisfies:

(1) For all $x \in X$, the map $y \rightarrow\{x x y\}$ from $X$ to $X$ is a hermitian operator on $X$ and has nonnegative spectrum.

(2) $\{a b\{x y z\}\}=\{\{a b x\} y z\}-\{x\{b a y\} z\}+\{x y\{a b z\}\}$ for all $a, b, x, y, z \in X$.

(3) $\|\{x x x\}\|=\|x\|^{3}$ for all $x \in X$.

We also recall that a bounded linear operator $T$ on a complex Banach space $X$ is said to be hermitian if $\|\exp (i r T)\|=1$ for every real number $r$.

Following [14, we define real $J B^{*}$-triples as norm-closed real subtriples of complex $J B^{*}$-triples. Here, by a subtriple we mean a subspace which is closed under triple products of its elements. A triple ideal of a real or complex $J B^{*}$-triple $X$ is a subspace $M$ of $X$ such that $\{X X M\}+\{X M X\} \subseteq M$. Real $J B W^{*}$-triples where first introduced as those real $J B^{*}$-triples which are dual Banach spaces in such a way that the triple product becomes separately $w^{*}$-continuous (see [14, Definition 4.1 and Theorem 4.4]). Later, it was shown in [18] that the requirement of separate $w^{*}$-continuity of the triple product is superabundant.

Finally, we recall that an element $x$ of a real $\mathrm{JB}^{*}$-triple $E$ is said to be a tripotent if $\{x x x\}=x$. Given $x, y$ tripotents in $E$, we say that $x$ and $y$ are orthogonal if $\{u u v\}=0$ and we say that $x \geq y$ if $x-y$ and $y$ are orthogonal tripotents. Then a minimal tripotent will be a tripotent which is minimal in the partial order defined above.

Examples of real $J B^{*}$-triples are the spaces $L(H, K)$, for arbitrary real, complex, or quaternionic Hilbert spaces $H$ and $K$, under the triple product $\{x y z\}:=$ $\frac{1}{2}\left(x y^{*} z+z y^{*} x\right)$. Also, the corresponding spaces of all symmetric, $S(H)$, and skew, $A(H)$, bounded linear operators on $H$ can be considered real $J B^{*}$-triples. The above examples become particular cases of those arising by considering either the so-called complex Cartan factors (regarded as real $J B^{*}$-triples) or real forms of complex Cartan factors [16]. We recall that real forms of a complex Banach space $X$ are defined as the real closed subspaces of $X$ of the form $X^{\tau}:=\{x \in X: \tau(x)=x\}$, for some conjugation (i.e., conjugate-linear isometry of period two) on $X$. We note that, if $X$ is a complex $J B^{*}$-triple, then every real form of $X$ is a real $J B^{*}$-triple (since conjugations on $X$ preserve triple products [15]). Among complex Cartan factors, the so-called complex spin factors become especially relevant for our present approach. They are built from an arbitrary complex Hilbert space $(H,(\cdot \mid \cdot))$ of hilbertian dimension $\geq 3$ by taking a conjugation $\sigma$ on $H$, and then by defining the triple product and the norm by

$$
\{x y z\}:=(x \mid y) z+(z \mid y) x-(x \mid \sigma(z)) \sigma(y)
$$

and

$$
\|x\|^{2}:=(x \mid x)+\sqrt{(x \mid x)^{2}-|(x \mid \sigma(x))|^{2}},
$$

respectively, for all $x, y, z$ in $H$. Following [19, we say that a real $J B^{*}$-triple is a generalized real spin factor if it is either a complex spin factor (regarded as a real $J B^{*}$-triple) or a real form of a complex spin factor.

It is well known that every complex $\mathrm{JBW}^{*}$-triple $E$ has a unique isometric predual $V$, and this is an L-embedded space. This is also the case for preduals of real $\mathrm{JBW}^{*}$-triples as done in [4].

Now we are ready to show the application of Theorem 2.8. 
Theorem 2.12. Let $A$ be a real $J B W^{*}$-triple and let $A_{*}$ be its predual. If $A$ is atomless, that is, A lacks minimal tripotentes, then every nonempty relatively weakly open subset of $B_{A_{*}}$ has diameter 2 .

Proof. As $A$ is atomless, according to [21, Corollary 2.1], we deduce that $B_{A_{*}}$ lacks extreme points. Now, $A_{*}$ is an L-embedded space by [4, Proposition 2.2]; then we have $A^{*}=A_{*} \oplus_{1} Z$, for some subspace $\mathrm{Z}$ of $A^{*}$. Let us see that $B_{Z}$ is weak*-dense in $B_{A^{*}}$.

As $B_{A_{*}}$ lacks extreme points and the set of extreme points of $B_{A^{*}}$ is the union of the sets of extreme point of $B_{A_{*}}$ and $B_{Z}$, we obtain that $\operatorname{ext}\left(B_{A^{*}}\right)=\operatorname{ext}\left(B_{Z}\right)$, where $\operatorname{ext}(K)$ denotes the set of extreme points of $K$. Now, the Krein-Milman theorem applied to $B_{A^{*}}$ gives us that $B_{A^{*}}=\overline{c o}^{w^{*}}\left(\operatorname{ext}\left(B_{Z}\right)\right)$, and then the desired conclusion.

Finally, it is enough to apply Theorem 2.8 to finish the proof.

In the setting of $C^{*}$-algebras, the concept of mininal tripotents is exactly the well-known notion of minimal projections. As a Von Neumann algebra is also a real $\mathrm{JBW}^{*}$-triple, we obtain the following

Corollary 2.13. Let $A$ be an atomless Von Neumann algebra, that is, A lacks minimal projections, and $A_{*}$ stands for its predual. Then every nonempty relatively weakly open subset of $B_{A_{*}}$ has diameter 2 .

Finally, we show a characterization of Radon-Nikodym property in the setting of the preduals of real $J B W^{*}$-triples and, as a consequence, in the setting of the preduals of Von Neumann algebras, too.

Theorem 2.14. Let $A$ be real $J B W^{*}$-triple and $A_{*}$ its predual. Then:

i) $A_{*}$ satisfies the Radon-Nikodym property if, and only if, $A$ is purely atomic, that is, $A$ is the weak-* closed linear span of its minimal tripotents. Furthermore, in this case, $A$ is the $\ell_{\infty}$-sum of weak-* closed simple ideals which are either finite-dimensional, infinite-dimensional generalized real spin factors or of the form $L(H, K), S(H)$ or $A(H)$ for some real, complex or quaternionic infinite-dimensional Hilbert spaces $H$ and $K$.

ii) $A_{*}$ fails the Radon-Nikodym property if, and only if, $A_{*}$ can be equivalently renormed so that every nonempty relatively weakly open subset of $B_{A_{*}}$ has diameter 2 .

iii) $A_{*}$ satisfies the Radon-Nikodym property if, and only if, $A_{*}$ satisfies the Krein-Milman property.

Proof. i) Assume that $A_{*}$ verifies the Radon-Nikodym property. By 21, Theorem 3.6], we have $A=B \oplus_{\infty} C$, where $B$ and $C$ are weak* closed real triple ideals of $A$, such that $B$ is purely atomic and $C$ is atomless. By the above corollary $C=0$, since the Radon-Nikodym property is hereditary. Then $A=B$ is purely atomic.

Now, in order to describe the preduals of real JBW* triples satisfiyng the RadonNikodym property, assume that $A$ is purely atomic. We denote by $\hat{A}=A \oplus i A$ the complexification of $A$. By [21, $\hat{A}$ is a purely atomic complex JBW* triple and then, by [11], $\hat{A}$ is the $\ell_{\infty}$ sum of type I Cartan factors, that is, the $\ell_{\infty}$ sum of $\mathrm{w}^{*}$-closed simple ideals which are either finite-dimensional, infinite-dimensional complex spin factors or of the form $L(H, K), S(H)$ or $A(H)$ for some complex Hilbert spaces $H$ and $K$. Taking into account that the conjugation $\tau$ preserves the triple product 
and is $\mathrm{w}^{*}$-continuous, it is enough to apply [16] to deduce that $A$ is the $\ell_{\infty}$ sum of $\mathrm{w}^{*}$-closed simple ideals which are either finite-dimensional, infinite-dimensional generalized real spin factors or of the form $L(H, K), S(H)$ or $A(H)$ for some real, complex or quaternionnic Hilbert spaces $H$ and $K$. Finally, as the Radon-Nikodym property is stable by $\ell_{1}$ sums and the preduals of the above spaces satisfy the Radon-Nikodym property (see [7]) we deduce that $A_{*}$ verifies the Radon-Nikodym property.

ii) If $A_{*}$ fails the Radon-Nikodym property, as in the above paragraph, we set $A=B \oplus_{\infty} C$. Now, as $B$ and $C$ are $w^{*}$-closed real triple ideals of $A$, we have $A_{*}=D \oplus_{1} E$, where $E$ is the predual of the atomless real $J B W^{*}$-triple $C$. By the above corollary, every nonempty relatively weakly open subset of $B_{E}$ has diameter 2 . Now it is enough to apply Lemma 2.1, to see that $A_{*}$ can be equivalently renormed so that every nonempty relatively weakly open subset of $B_{A_{*}}$ has diameter 2 . The converse implication is trivial.

iii) It is well known that every Banach space satisfying the Radon-Nikodym property also verifies the Krein-Milman property. In order to prove the converse, assume that $A_{*}$ fails the Radon-Nikodym property. Then, by i), $A$ is not purely atomic. Now, as in ii), $A=B \oplus_{\infty} C$, where $B$ and $C \neq 0$ are weak* closed real triple ideals of $A$, such that $B$ is purely atomic and $C$ is atomless. Then, $A_{*}=D \oplus_{1} E$, where $E$ is the predual of the atomless real $J B W^{*}$-triple $C$. By [21, Corollary 2.1 , we deduce that $B_{E}$ lacks extreme points and hence $A_{*}$ fails the Krein-Milman property.

Corollary 2.15. Let $A$ be a Von Neumann algebra and $A_{*}$ its predual. Then:

i) $A_{*}$ satisfies the Radon-Nikodym property if, and only if, $A$ is purely atomic. Furthermore, in this case, there exists $\left\{H_{i}\right\}$ a family of infinite-dimensional complex Hilbert spaces, such that $A=\ell_{\infty}-\sum_{i} L\left(H_{i}\right)$ and $A_{*}=\ell_{1}-$ $\sum_{i} N\left(H_{i}\right)$, where $N(H)$ denotes the space of all nuclear operators on $H$.

ii) $A_{*}$ fails the Radon-Nikodym property if, and only if, $A_{*}$ can be equivalently renormed so that every nonempty relatively weakly open subset of $B_{A_{*}}$ has diameter 2 .

iii) $A_{*}$ satisfies the Radon-Nikodym property if, and only if, $A_{*}$ satisfies the Krein-Milman property.

\section{ACKNOWLEGMENTS}

This work was finished when the author visited the Department of Mathematics of the University of Texas at Austin, supported by a grant from Programa de Sabáticos, Junta de Andalucía (Spain). I thank Professor Haskell Rosenthal for his kindness and his fruitful conversations during this stay. Also I thank the referee for his comments which improved the final version of this paper.

\section{REFERENCES}

[1] T. Andô. On the predual of $H^{\infty}$. Commentationes Mathematicae. Special volume in honour of W. Orlicz. (1978), 33-40. MR0504151 (80c:46063)

[2] T. Barton and G. Godefroy. Remarks on the predual of a JB*-triple. J. London Math. Soc. (2) 34, (1986), 300-304. MR0856513 (87i:46047)

[3] J. Becerra and G. López. Relatively weakly open subsets of the unit ball in functions spaces. Preprint. 
[4] J. Becerra, G. López, A. Peralta and A. Rodríguez. Relatively weakly open sets in closed balls of Banach spaces, and real JB*-triples of finite rank. Mat. Ann. 330, (2004), 45-58. MR2091678 (2005f:46128)

[5] J. Becerra, G. López and A. Rodríguez. Relatively weakly open sets in closed balls of $C^{*}$ algebras. J. London Math. Soc. (2) 68, (2003), 753-761. MR2010009 (2004i:46082)

[6] Bor-Luh Lin, Pei-Kee Lin and S. L. Troyanski. Characterizations of denting points. Proc. Amer. Math. Soc. 102, (1988), 526-528. MR0928972 (89e:46016)

[7] Cho-Ho Chu. On the Radon-Nikodym property in Jordan algebras. Glasgow Math. J. 24, (1983), 185-189. MR0706149 (84i:46070)

[8] Cho-Ho Chu and B. Iochum. Remarks on the Radon-Nikodym property in Jordan triples. Proc. Amer. Math. Soc. 99, (1987), no. 3, 462-464. MR0875381(88a:46080)

[9] Cho-Ho Chu. A note on scattered $C^{*}$-algebras and the Radon-Nikodym property. J. London Math. Soc. 24, (1981), 533-536. MR0635884 (82k:46086)

[10] R. Deville, G. Godefroy and V. Zizler. Smoothness and renormings in Banach spaces. Pitman Monographs and Surveys in Pure and Applied Math. 64, 1993. MR1211634 (94d:46012)

[11] Y. Friedman and B. Russo. Structure of the predual of a JBW*-triple. J. Reine Angew. Math. 356, (1985), 67-89. MR0779376 (86f:46073)

[12] P. Harmand, D. Werner and W. Werner. M-Ideals in Banach spaces and Banach algebras. Lecture Notes in Math, 1547, Springer-Verlag, Berlin, 1993. MR1238713 (94k:46022)

[13] Z. Hu and M. A. Smith. On the extremal structure of the unit balls of Banach spaces of weakly continuous functions and their duals. Trans. Amer. Math. Soc. 349, (1997), 19011918. MR.1407701 (97h:46054)

[14] J. M. Isidro, W. Kaup, and A. Rodríguez. On real forms of $J B^{*}$-triples. Manuscripta Math. 86, (1995), 311-335. MR1323795 (96a:46121)

[15] W. Kaup. A Riemann mapping theorem for bounded symmetric domains in complex Banach spaces. Math. Z. 183, (1983), 503-529. MR0710768 (85c:46040)

[16] W. Kaup. On real Cartan factors. Manuscripta Math. 92, (1997), 191-222. MR.1428648 (97m:46109)

[17] D. Li. Espaces L-facteurs de leurs biduaux: bonne disposition, meilleure approximation et Propriété de Radon-Nikodym. Quart. J. Math. Oxford (2), 38, (1987), 229-243. MR0891618 (88h:46024)

[18] J. Martínez and A. M. Peralta. Separate weak*-continuity of the triple product in dual real JB*-triples. Math. Z. 234, 635-646. MR.1778403 (2001h:46123)

[19] A. Moreno and A. Rodríguez. On the Zel'manovian classification of prime JB*-triples. J. Algebra 226, (2000), 577-613. MR:1749905 (2001c:46122)

[20] O. Nygaard and D. Werner. Slices in the unit ball of a uniform algebra. Archiv Math. 76, (2001), 441-444. MR1831500 (2002e:46057)

[21] A. Peralta and L. L. Stachó. Atomic decomposition of real JBW*-triples. Quart. J. Math. 52, (2001), 79-87. MR 1820904 (2001m:46154)

[22] D. Werner. M-Ideals and the Basic Inequality. J. Approx. Theory 76, (1994), 21-30. MR.1257062 (95i:47080)

[23] T. S. S. R. K. Rao. There are no denting points in the unit ball of $W C(K, X)$. Proc. Amer. Math. Soc. 127, (1999), 2969-2973. MR1610781(2000a:46056)

Facultad de Ciencias, Departamento de Análisis Matemático, Universidad de GraNADA, 18071-GRANADA, SPAIN

E-mail address: glopezp@ugr.es 